\title{
Medial Axis LUT Computation for Chamfer Norms Using $\mathcal{H}$-Polytopes
}

\author{
Nicolas Normand and Pierre Évenou \\ IRCCyN UMR CNRS 6597, École polytechnique de l'Université de Nantes, \\ Rue Christian Pauc, La Chantrerie, \\ 44306 Nantes Cedex 3, France \\ Nicolas. Normand@polytech. univ-nantes.fr, \\ Pierre.Evenou@polytech.univ-nantes.fr
}

\begin{abstract}
Chamfer distances are discrete distances based on the propagation of local distances, or weights defined in a mask. The medial axis, i.e. the centers of the maximal disks (disks which are not contained in any other disk), is a powerful tool for shape representation and analysis. The extraction of maximal disks is performed in the general case with comparison tests involving look-up tables representing the covering relation of disks in a local neighborhood. Although look-up table values can be computed efficiently 1, the computation of the look-up table neighborhood tend to be very time-consuming. By using polytope [2] descriptions of the chamfer disks, the necessary operations to extract the look-up tables are greatly reduced.
\end{abstract}

\section{Introduction}

The distance transform $D T_{X}$ of a binary image $X$ is a function that maps each point $x$ with its distance to the background i.e. with the radius of the largest open disk centered in $x$ included in the image. Such a disk is said to be maximal if no other disk included in $X$ contains it. The set of centers of maximal disks, the medial axis, is a convenient description of binary images for many applications ranging from image coding to shape recognition. Its attractive properties are reversibility and (relative) compactness.

Algorithms for computing the distance transform are known for various discrete distances 3456. In this paper, we will focus on chamfer (or weighted) distances. The classical medial axis extraction method is based on the removal of non maximal disks in the distance transform. It is thus mandatory to describe the covering relation of disks, or at least the transitive reduction of this relation. For simple distances this knowledge is summarized in a local maximum criterion 3. The most general method for chamfer distances uses look-up tables for that purpose [7].

In this paper we propose a method to both compute the look-up tables and the look-up table mask based on geometric properties of the balls of chamfer norms. Basic notions, definitions and known results about chamfer disks and medial 
axis look-up tables are recalled in section [2. Then section 3 justifies the use of polytope formalism in our context and presents the principles of the method. In section 4, algorithms for the $2 \mathrm{D}$ case are given.

\section{Chamfer Medial Axis}

\subsection{Chamfer Distances}

Definition 1 (Discrete distance, metric and norm). Consider a function $d: \mathbb{Z}^{n} \times \mathbb{Z}^{n} \rightarrow \mathbb{N}$ and the following properties $\forall x, y, z \in \mathbb{Z}^{n}, \forall \lambda \in \mathbb{Z}$ :

1. positive definiteness $d(x, y) \geq 0$ and $d(x, y)=0 \Leftrightarrow x=y$,

2. symmetry $d(x, y)=d(y, x)$,

3. triangle inequality $d(x, z) \leq d(x, y)+d(y, z)$,

4. translation invariance $d(x+z, y+z)=d(x, y)$,

5. positive homogeneity $d(\lambda x, \lambda y)=|\lambda| \cdot d(x, y)$.

$d$ is called a distance if it verifies conditions 1 and 2, a metric with conditions 1 to 3 and $a$ norm if it also satisfies conditions 4 and 5 .

Most discrete distances are built from a definition of neighborhood and connected paths (path-generated distances), the distance from $x$ to $y$ being equal to the length of the shortest path between the two points 8 . Distance functions differ by the way path lengths are measured: as the number of displacements in the path for simple distances like $d_{4}$ and $d_{8}$, as a weighted sum of displacements for chamfer distances 4 or by the displacements allowed at each step for neighborhood sequence distances [8, , or even by a mixed approach of weighted neighborhood sequence paths [6].

For a given distance $d$, the closed ball $B^{c}$ and open ball $B^{o}$ of center $c$ and radius $r$ are the sets of points of $\mathbb{Z}^{n}$ :

$$
\begin{aligned}
& B^{o}(c, r)=\{p: d(c, p)<r\} \\
& B^{c}(c, r)=\{p: d(c, p) \leq r\}
\end{aligned}
$$

Since the codomain of $d$ is $\mathbb{N}: \forall r \in \mathbb{N}, d(c, p) \leq r \Leftrightarrow d(c, p)<r+1$. So:

$$
\forall r \in \mathbb{N}, B^{c}(c, r)=B^{o}(c, r+1)
$$

In the following, the notation $B$ will be used to refer to closed balls.

Definition 2 (Chamfer mask [9]). A weighting $M=(\vec{v} ; w)$ is a vector $\vec{v}$ of $\mathbb{Z}^{n}$ associated with a weight $w$ (or local distance). A chamfer mask $\mathcal{M}$ is a central-symmetric set of weightings having positive weights and non-null displacements, and containing at least one basis of $\mathbb{Z}^{n}: \mathcal{M}=\left\{M_{i} \in \mathbb{Z}^{n} \times \mathbb{N}^{*}\right\}_{1 \leq i \leq m}$

The grid $\mathbb{Z}^{n}$ is symmetric with respect to the hyperplanes normal to the axes and to the bisectors (G-symmetry). This divides $\mathbb{Z}^{n}$ in $2^{n} . n$ ! sub-spaces ( 8 octants 
for $\mathbb{Z}^{2}$ ). Chamfer masks are usually G-symmetric so that weightings may only be given in the sub-space $0 \leq x_{n} \leq \ldots \leq x_{1}$.

Paths between two points $x$ and $y$ can be produced by chaining displacements. The length of a path is the sum of the weights associated with the displacements and the distance between $x$ and $y$ is the length of the shortest path.

Definition 3 (Chamfer distance [9]). Let $\mathcal{M}=\left\{\left(\overrightarrow{v_{i}}, w_{i}\right) \in \mathbb{Z}^{n} \times \mathbb{N}^{*}\right\}_{1 \leq i \leq m}$ be a chamfer mask. The chamfer (or weighted) distance between two points $x$ and $y$ is:

$$
d(x, y)=\min \left\{\sum \lambda_{i} w_{i}: x+\sum \lambda_{i} \overrightarrow{v_{i}}=y, \lambda_{i} \in \mathbb{N}, 1 \leq i \leq m\right\} .
$$

Any chamfer masks define a metric [10. However a chamfer mask only generates a norm when some conditions on the mask neighbors and on the corresponding weights permits a triangulation of the ball in influence cones 911. When a mask defines a norm then all its balls are convex.

\subsection{Geometry of the Chamfer Ball}

We can deduce from (1) and (3) a recursive construction of chamfer balls:

$$
B(O, r)=B(O, r-1) \cup \bigcup_{0 \leq i \leq m} B\left(O+\overrightarrow{v_{i}}, r-w_{i}\right)
$$

Definition 4 (Influence cone [12]). Let $\mathcal{M}=\left\{\left(\overrightarrow{v_{i}}, w_{i}\right) \in \mathbb{Z}^{n} \times \mathbb{N}^{*}\right\}_{1 \leq i \leq m}$ be a chamfer mask generating a norm. An influence cone is a cone from the origin spanned by a subset of the mask vectors $\left\{\overrightarrow{v_{i}}, \overrightarrow{v_{j}}, \overrightarrow{v_{k}}, \ldots\right\}$ in which only the weightings $M_{i}, M_{j}, M_{k}, \ldots$ of the mask are involved in the computation of the distance from $O$ to any point of the cone.

In each influence cone, the discrete gradient of the distance function is constant and equal to 9]:

$$
\left(w_{i}, w_{j}, w_{k}, \ldots\right) \cdot\left(\overrightarrow{v_{i}}\left|\overrightarrow{v_{j}}\right| \overrightarrow{v_{k}} \mid \ldots\right)^{-1}
$$

where $\left(\overrightarrow{v_{i}}\left|\overrightarrow{v_{j}}\right| \overrightarrow{v_{k}} \mid \ldots\right)$ stands for the column matrix of the vectors spanning the cone. The distance $d_{C}(O, p)$ from the origin to any point $p$ of this cone $C$ is then:

$$
d_{C}(O, p)=\left(w_{i}, w_{j}, w_{k}, \ldots\right) \cdot\left(\overrightarrow{v_{i}}\left|\overrightarrow{v_{j}}\right| \overrightarrow{v_{k}} \mid \ldots\right)^{-1} \cdot p
$$

For instance, with chamfer norm $d_{5,7,11}$, the point $(3,1)$ is in the cone spanned by the vectors $a=(1,0)$ and $c=(2,1)$ and the weights involved are 5 and 11 . The distance between the origin and the point $(3,1)$ is then:

$$
d_{C_{a, c}}(O,(3,1))=\left(\begin{array}{ll}
5 & 11
\end{array}\right) \cdot\left(\begin{array}{ll}
1 & 2 \\
0 & 1
\end{array}\right)^{-1} \cdot\left(\begin{array}{l}
3 \\
1
\end{array}\right)=\left(\begin{array}{ll}
5 & 1
\end{array}\right) \cdot\left(\begin{array}{l}
3 \\
1
\end{array}\right)=16 .
$$




\subsection{Chamfer Medial Axis}

For simple distances $d_{4}$ and $d_{8}$, the medial axis extraction can be performed by the detection of local maxima in the distance map [13. Chamfer distances raise a first complication even for small masks as soon as the weights are not unitary. Since all possible values of distance are not achievable, two different radii $r$ and $r^{\prime}$ may correspond to the same set of discrete points. The radii $r$ and $r^{\prime}$ are said to be equivalent. Since the distance transform labels pixels with the greatest equivalent radius, criterions based on radius difference fail to recognize equivalent disks as being covered by other disks. In the case of $3 \times 32 \mathrm{D}$ masks or $3 \times 3 \times 33$ D masks, a simple relabeling of distance map values with the smallest equivalent radius is sufficient [1415. However this method fails for greater masks and the most general method for medial axis extraction from the distance map involves look-up tables (LUT) that represent for each radius $r$ and displacement $\overrightarrow{v_{i}}$, the minimal open ball covering $B^{\circ}\left(O, r_{1},\right)$ in direction $\overrightarrow{v_{i}} \quad\left[\begin{array}{l}\text { : } \\ \text { : }\end{array}\right.$

$$
\text { Lut }_{v_{i}}\left(r_{1}\right)=\min \left\{r_{2}: B^{o}\left(O+\overrightarrow{v_{i}}, r_{1}\right) \subseteq B^{o}\left(O, r_{2}\right)\right\} .
$$

Equivalently using closed balls (considering (2)):

$$
\text { Lut }_{\overrightarrow{v_{i}}}\left(r_{1}\right)=1+\min \left\{r_{2}: B\left(O+\overrightarrow{v_{i}}, r_{1}-1\right) \subseteq B\left(O, r_{2}\right)\right\} .
$$

Consider for instance the $d_{5,7,11}$ distance [16, Fig. 14]. $\operatorname{Lut}_{(1,0)}(10)=12$ means that $B^{o}(O, 10) \subseteq B^{o}((2,1), 12)$ but $B^{o}(O, 10) \nsubseteq B^{o}((2,1), 11)$. Or, in terms of closed balls, $B(O, 9) \subseteq B((2,1), 11)$ but $B(O, 9) \nsubseteq B((2,1), 10)$.

Medial Axis LUT Coefficients. A general method for LUT coefficient computation was given by Rémy and Thiel [12/17/9. The idea is that the disk covering relation can be extracted directly from values of distance to the origin. If $d(O, p)=r_{1}$ and $d\left(O, p+\overrightarrow{v_{i}}\right)=r_{2}$, we can deduce the following:

$$
\begin{aligned}
& p \in B\left(O, r_{1}\right)=B^{o}\left(O, r_{1}+1\right), \\
& p+\overrightarrow{v_{i}} \notin B\left(O, r_{2}-1\right)=B^{o}\left(O, r_{2}\right),
\end{aligned}
$$

hence $B^{o}\left(O+\overrightarrow{v_{i}}, r_{1}+1\right) \not \subset B^{o}\left(O, r_{2}\right)$ and Lut $\overrightarrow{v_{i}}\left(r_{1}+1\right)>r_{2}$. If $\forall p, d(O, p) \leq$ $r_{1} \Rightarrow d\left(O, p+\overrightarrow{v_{i}}\right) \leq r_{2}$ then Lut $\overrightarrow{v_{i}}\left(r_{1}+1\right)=r_{2}+1$.

Finally: Lut $\overrightarrow{v_{i}}(r)=1+\max \left(d\left(O, p+\overrightarrow{v_{i}}\right): d(O, p)<r\right)$.

This method only requires one scan of the distance function for each displacement $\overrightarrow{v_{i}}$. Moreover, the visited area may be restricted according to the symmetries of the chamfer mask. The order of complexity is about $O\left(m L^{n}\right)$ if we limit the computation of the distance function to a $L^{n}$ image.

Medial Axis LUT Mask. Thiel observed that the chamfer mask is not adequate to compute the LUT and introduced a LUT Mask $\mathcal{M}_{\text {Lut }}(R)$ for that purpose [12, p. 81]. $\mathcal{M}_{\text {Lut }}(R)$ is the minimal test neighbourhood sufficient to detect the medial axis for shapes whose inner radius (the radius of a greatest ball) is less than or equal to $R$. For instance, with $d_{14,20,31,44}: \operatorname{Lut}_{(2,1)}(291)=$ 
321 and $\operatorname{Lut}_{(2,1)}(321)=352$ but the smallest open ball of center $\mathrm{O}$ covering $B^{o}((4,2), 291)$ is $B^{o}(O, 351)$. In this particular case, the point $(4,2)$ is not in the chamfer mask but should be in $\mathcal{M}_{\text {Lut }}(R)$ for $R$ greater than 350 .

A mask incompleteness produces extra points in the medial axis (undetected ball coverings). A general method for both detecting and validating $\mathcal{M}_{\text {Lut }}$ is based on the computation of the medial axis of all disks. When $\mathcal{M}_{\text {Lut }}$ is complete, the medial axis is restricted to the center of the disk, when extra points remains, they are added to $\mathcal{M}_{\text {Lut }}$. This neighborhood determination was proven to work in any dimension $n \geq 2$. However it is time consuming even when taking advantage of the mask symmetries.

\section{Method Basics}

\subsection{General $\mathcal{H}$-Polytopes [2]}

Definition 5 (Polyhedron). A convex polyhedron is the intersection of a finite set of half-hyperplanes.

Definition 6 (Polytope). A polytope is the convex hull of a finite set of points.

Theorem 1 (Weyl-Minkowski). A subset of Euclidean space is a polytope if and only if it is a bounded convex polyhedron.

As a result, a polytope in $\mathbb{R}^{n}$ can be represented either as the convex hull of its $k$ vertices $\left(\mathcal{V}\right.$-description): $P=\operatorname{conv}\left(\left\{p_{i}\right\}_{1 \leq i \leq k}\right)$ or by a set of $l$ half-planes $(\mathcal{H}$-description):

$$
P=\{x: A x \leq y\},
$$

where $A$ is a $l \times n$ matrix, $y$ a vector of $n$ values that we name $\mathcal{H}$-coefficients of $P$. Having two vectors $\vec{u}$ and $\vec{v}$, we denote $\vec{u} \leq \vec{v}$ if and only if $\forall i, \vec{u}_{i} \leq \vec{v}_{i}$.

Definition 7 (Discrete polytope). A discret polytope is the intersection of a polytope with $\mathbb{Z}^{n}$.

Minimal Representation. Many operations on $\mathbb{R}^{n}$ polytopes in either $\mathcal{V}$ or $\mathcal{H}$ representation often require a minimal representation. The redundancy removal is the removing of unnecessary vertices or inequalities in polytopes. Since our purpose is mainly to compare $\mathcal{H}$-polytopes defined with the same matrix $A$, no inequality removal is needed. However, for some operations, $\mathcal{H}$-representations of discrete polytopes must be minimal in terms of $\mathcal{H}$-coefficients.

Definition 8 (Minimal parameter representation). We call minimal parameter $\mathcal{H}$-representation of a discrete polytope $P$, denoted $\widehat{\mathcal{H}}$-representation, a $\mathcal{H}$-representation $P=\{x: A x \leq y\}$ such that $y$ is minimal:

$$
P=\left\{x \in \mathbb{Z}^{n}: A x \leq y\right\} \text { and } \forall i \in[1 . . l], \exists x \in \mathbb{Z}^{n}: A_{i} x=y_{i},
$$

where $A_{i}$ means line $i$ in matrix $A$. 


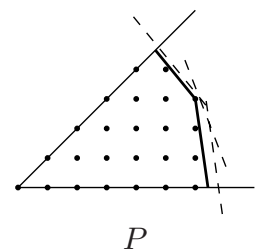

$$
\left(\begin{array}{ll}
14 & 2 \\
13 & 5 \\
11 & 9
\end{array}\right)
$$

$A$

$$
\left(\begin{array}{l}
95 \\
95 \\
95
\end{array}\right)
$$

$y$

$$
\left(\begin{array}{l}
90 \\
93 \\
93
\end{array}\right)
$$

$\widehat{\mathcal{H}}(P)$

Fig. 1. $\mathcal{H}$-representations of a discrete G-symmetrical polytope $P$ (restricted to the first octant). Dashed lines: $\mathcal{H}$-representation of $P$. Thick lines: $\widehat{\mathcal{H}}$-representation of $P$. In the $\widehat{\mathcal{H}}$ case, the three equalities are verified for the same point $(6,3)$. Notice that although coefficient values are minimal, this representation is still redundant: the second inequality could be removed.

The $\widehat{\mathcal{H}}$ function which gives the minimal parameters for a given polytope $P$ is introduced for convenience: $\widehat{\mathcal{H}}(P)=\max \{A x: x \in P\}$. $\{x: A x \leq \widehat{\mathcal{H}}(P)\}$ is the $\widehat{\mathcal{H}}$-representation of $P=\{x: A x \leq y\}$. Figure 1 depicts two representations of the same polytope $P$ in $\mathbb{Z}^{2}$.

$\mathcal{H}$-Polytope Translation. Let $P=\{x: A x \leq y\}$ be a $\mathcal{H}$-polytope. The translated of $P$ by $\vec{v}$ which is also the Minkowski sum of $P$ and $\{\vec{v}\}$ is:

$$
(P)_{\vec{v}}=P \oplus\{\vec{v}\}=\{x+\vec{v}: A x \leq y\}=\{x: A x \leq y+A \vec{v}\} .
$$

The translation of a minimal representation gives a minimal representation.

Covering Test. Let $P=\{x: A x \leq y\}$ and $Q=\{x: A x \leq z\}$ be two polyhedrons represented by the same matrix $A$ but different sets of $\mathcal{H}$-coefficients $y$ and $z . P$ is a subset of $Q$ if (sufficient condition):

$$
y \leq z \Rightarrow P \subseteq Q .
$$

Furthermore, if the $\mathcal{H}$-description of the enclosed polyhedron has minimal coefficients, the condition is also necessary:

$$
y=\widehat{\mathcal{H}}(P) \leq z \Leftrightarrow P \subseteq Q .
$$

\subsection{Chamfer $\mathcal{H}$-Polytopes}

Describing balls of chamfer norms as polygons in 2D and polyhedra in higher dimensions is not new [10. Thiel and others have extensively studied chamfer ball geometry from this point of view [12]11/18. Our purpose is to introduce properties specific to the $\mathcal{H}$-representation of these convex balls.

\section{Proposition 1 (Direct distance formulation)}

$$
d(O, x)=\max _{1 \leq i \leq l}\left(d_{C_{i}}(x)\right)
$$

where $l$ is the number of cones, $d_{C_{i}}$ is the distance function in the $i^{t h}$ cone. Note that this formula does not require to determine in which cone lies $x$. 
Proof. Equation (6) states that for all the influence cones $C$ that contain $p$, $d_{C}(O, p)=d(O, p)$. For other cones, this relation does not hold, but it is still possible to compute $d_{C}(O, p)$. The influence cone $C$ corresponds to a facet of the unitary real ball supported by the hyperplane $\left\{x \in \mathbb{R}^{n}: d_{C}(x)=1\right\}$. Due to convexity, the unitary ball is included in the halfspace $\left\{x \in \mathbb{R}^{n}: d_{C}(x) \leq 1\right\}$. This applies to the vertices of the unitary ball $\frac{\overrightarrow{v_{i}}}{w_{i}}: d_{C}\left(\frac{\overrightarrow{v_{i}}}{w_{i}}\right) \leq 1$. By linearity of $d_{C}, d_{C}\left(\overrightarrow{v_{i}}\right) \leq w_{i}$ and $d_{C}\left(\sum_{i} \lambda_{i} \overrightarrow{v_{i}}\right)=\sum_{i} \lambda_{i} d_{C}\left(\overrightarrow{v_{i}}\right) \leq \sum_{i} \lambda_{i} w_{i} \cdot d_{C}\left(\sum_{i} \lambda_{i} \overrightarrow{v_{i}}\right)$ is always less than or equal to the length of the path $\sum_{i} \lambda_{i} \overrightarrow{v_{i}}$.

Chamfer Balls $\mathcal{H}$-Representation. The $\mathcal{H}$-representation of chamfer balls is directly derived from (13):

$$
x \in B(O, r) \Leftrightarrow \max _{1 \leq i \leq l}\left\{d_{C_{i}}(x)\right\} \leq r \Leftrightarrow A_{\mathcal{M}} \cdot x \leq y
$$

where $A_{\mathcal{M}}$ is a $\mathcal{H}$-representation matrix depending only on the chamfer mask $\mathcal{M}$. The number of rows in $A_{\mathcal{M}}$ is equal to the number $l$ of influence cones, each line of the matrix $A_{\mathcal{M}}$ is computed with (5) and $y$ is a column vector whose values are $r$. For instance, the $\mathcal{H}$-representation matrix for $d_{5,7,11}$ is $A_{\mathcal{M}}=\left(\begin{array}{ll}5 & 1 \\ 4 & 3\end{array}\right)$ where $\left(\begin{array}{ll}5 & 1\end{array}\right)$ and $\left(\begin{array}{lll}4 & 3\end{array}\right)$ are the distance gradients in the two cones and $B(O, r)=\left\{x \in \mathbb{Z}^{n}:\left(\begin{array}{ll}4 & 3 \\ 5 & 1\end{array}\right) \cdot x \leq\left(\begin{array}{l}r \\ r\end{array}\right)\right\}$.

Proposition 2 (Furthest point). Let $A_{\mathcal{M}}$ be the matrix defined by the chamfer mask $\mathcal{M}$ generating a norm. The furthest point from the origin in the $\widehat{\mathcal{H}}$ polytope $P=\left\{x: A_{\mathcal{M}} \cdot x \leq y\right\}$ is at a distance equal to the greatest component of $y$.

Proof. By construction of $A_{\mathcal{M}}$, (13) is equivalent to $d(O, x)=\max _{i}\left\{A_{\mathcal{M} i} \cdot x\right\}$.

$$
\max _{x \in P}\{d(O, x)\}=\max _{x \in P}\left\{\max _{1 \leq i \leq l}\left\{A_{\mathcal{M} i} \cdot x\right\}\right\}=\max \left\{\widehat{\mathcal{H}}_{i}(P)\right\}
$$

Proposition 3 (Minimal covering ball). The radius of the minimal ball centered in $O$ that contains all points of a discrete $\widehat{\mathcal{H}}$-polytope $P$ represented by the matrix $A_{\mathcal{M}}$ and the vector $y$ is equal to the greatest component of $y$.

Proof. The smallest ball that covers the polytope $P$ must cover its furthest point from the origin.

$$
\min \{r \in \mathbb{N}: P \subseteq B(O, r)\}=\max _{x \in P}\{d(O, x)\}
$$

Note that if $P$ is not centered in $O$, the simplification due to symmetries do not hold and the full set of $\mathcal{H}$-coefficients is needed, unless we ensure that the $\mathcal{H}$-coefficents for the hyperplanes in the working sub-space are greater than $\mathcal{H}$ coefficients for the corresponding symmetric cones. This is the case when a Gsymmetric polytope is translated by a vector in the sub-space. 
Definition 9 (Covering function). We call covering function of a set $X$ of points of $\mathbb{Z}^{n}$ the function $\mathcal{C}_{X}$ which assigns to each point $p$ of $\mathbb{Z}^{n}$, the radius of the minimal ball centered in $p$ covering $X$ :

$$
\begin{aligned}
\mathcal{C}_{X}: 2^{\mathbb{Z}^{n}} \times \mathbb{Z}^{n} & \rightarrow \mathbb{N} \\
X, p & \rightarrow \min \{r: X \subseteq B(p, r)\}
\end{aligned}
$$

The covering function of the chamfer ball $B(O, r)$ at point $p$ gives the radius of the minimal ball centered in $p$ that contains $B(O, r)$. It is equal using central symmetry to the minimal ball centered in $O$ covering $B(p, r)$ and therefore it is the maximal component of the $\widehat{\mathcal{H}}$-representation of $B(p, r)$ :

$$
\mathcal{C}_{B(O, r)}(p)=\max \left\{\widehat{\mathcal{H}}(B(p, r)\}=\max \left\{\widehat{\mathcal{H}}(B(O, r))+A_{\mathcal{M}} \cdot p\right\}\right.
$$

One can notice that the covering function of the zero radius disk is equal to the distance function, as is the distance transform of the complement of this disk:

$$
\mathcal{C}_{B(O, 0)}(p)=D T_{\mathbb{Z}^{n} \backslash\{O\}}(p)=d(p, O)=d(O, p) .
$$

Definition 10 (Covering cone). A covering cone $C_{o, U}$ in $\mathcal{C}_{X}$ is a cone defined by a vertex $o$ and a subset $U$ of the chamfer mask neighbor set with $\operatorname{det}(U)= \pm 1$, $C_{o, U}=\left\{o+\sum \lambda_{i} \overrightarrow{u_{i}}, \lambda_{i} \in \mathbb{N}, \overrightarrow{u_{i}} \in U\right\}$, such that:

$$
\forall p \in C_{o, U}, \forall \vec{u} \in U, B\left(p, \mathcal{C}_{X}(p)\right) \subsetneq B\left(p+\vec{u}, \mathcal{C}_{X}(p+\vec{u})\right) .
$$

Proposition 4. If $C_{o, U}$ is a covering cone in $\mathcal{C}_{B(O, r)}$ then for any point $q$ in $C_{o, U} \backslash U \backslash\{o\}$ there exists $p$ such that:

$$
B(O, r) \subsetneq B\left(p, \mathcal{C}_{B(O, r)}(p)\right) \subsetneq B\left(q, \mathcal{C}_{B(O, r)}(q)\right)
$$

Proof. Since $q \neq o$, there always exists another point $p$ in $C_{o, U}$, distinct from $q$, such that $B\left(p, \mathcal{C}_{B(O, r)}(p)\right) \subsetneq B\left(q, \mathcal{C}_{B(O, r)}(q)\right) . B(O, r) \subseteq B\left(p, \mathcal{C}_{B(O, r)}(p)\right)$ always holds by definition of $\mathcal{C}_{B(O, r)}$. A sufficient condition for $B(O, r) \neq B\left(p, \mathcal{C}_{B(O, r)}\right)$ is that a point $p$ distinct from $O$ exists in $C_{o, U}$ which is always the case if $q$ is not equal to one of the generating vectors of $C_{o, U}$.

Proposition 5. If there is a integer $j \in[1 \ldots l]$ and a point o such that $A_{\mathcal{M}_{j}}$. $\overrightarrow{u_{i}}, \forall \overrightarrow{u_{i}} \in U$ and $\widehat{\mathcal{H}}_{j}(B(o, r))$ are maximal then $C_{o, U}$ is a covering cone in $\mathcal{C}_{B(O, r)}$.

Proof. Let $j$ be the row number of a maximal component of $\widehat{\mathcal{H}}(B(o, r))$ and $A_{\mathcal{M}} \cdot \overrightarrow{u_{i}}, \forall \overrightarrow{u_{i}} \in U$, then $j$ is a maximal component of any positive linear combination of these vectors. Let $p$ be any point in $C_{o, U}, p=o+\sum_{i} \lambda_{i} \vec{u}_{i}, \lambda_{i} \in \mathbb{N}$ and $B_{p}$ the minimal ball covering $B(O, r)$. From (16) we deduce that $\mathcal{C}_{B(O, r)}$ is affine in $C_{o, U}$ :

$$
\begin{aligned}
\mathcal{C}_{B(O, r)}(p) & =\max \left\{\widehat{\mathcal{H}}(B(o, r))+\sum \lambda_{i} A_{\mathcal{M}} \overrightarrow{u_{i}}\right\} \\
& =\widehat{\mathcal{H}}_{j}(B(o, r))+\sum \lambda_{i} A_{\mathcal{M}} \vec{u}_{i}
\end{aligned}
$$


In the same way, the radius of the minimal ball centered in $p+\vec{u}, \vec{u} \in U$ covering $B(O, r)$ is:

$$
\begin{aligned}
\mathcal{C}_{B(O, r)}(p+\vec{u}) & =\widehat{\mathcal{H}}_{j}(B(o, r))+\sum \lambda_{i} A_{\mathcal{M}_{j}} \vec{u}_{i}+A_{\mathcal{M}_{j}} \vec{u} \\
& =\mathcal{C}_{B\left(p, \mathcal{C}_{B(O, r)}(p)\right)}(O+\vec{u}) .
\end{aligned}
$$

In other words, $B\left(p, \mathcal{C}_{B(O, r)}(p)\right)$ is a subset of $B\left(p+\vec{u}, \mathcal{C}_{B(O, r)}(p+\vec{u})\right)$.

\section{LUT and $\mathcal{M}_{\text {Lut }}$ Computation for 2D Chamfer Norms}

LUT and $\mathcal{M}_{\text {Lut }}$ computation methods for 2D chamfer norms are presented here. Both are based on the minimal $\widehat{\mathcal{H}}$-representation of the chamfer balls, from which we compute the covering function and deduce the LUT values. $\mathcal{M}$ vectors are ordered by angle so that each influence cone is defined by two successive angles $\left(O,\left\{\overrightarrow{v_{i}}, \overrightarrow{v_{i+1}}\right\}\right)$.

\section{1 $\widehat{\mathcal{H}}$-Representation of Chamfer Balls}

The computation of the LUT is based on a $\widehat{\mathcal{H}}$-representation of the chamfer norm balls. All share the same matrix $A_{\mathcal{M}}$ which depends only on the chamfer mask (14). $\widehat{\mathcal{H}}$-coefficients of balls are computed iteratively from the ball of radius 0 , $B(O, 0)=\left\{x: A_{\mathcal{M}} x=0\right\}$ using (4) and (10).

$$
\begin{aligned}
\widehat{\mathcal{H}}(B(O, r))=\max \{ & \widehat{\mathcal{H}}(B(O, r-1)), \\
& \left.\widehat{\mathcal{H}}\left(B\left(O, r-w_{1}\right)\right)+A_{\mathcal{M}} \overrightarrow{v_{1}}, \ldots, \widehat{\mathcal{H}}\left(B\left(O, r-w_{m}\right)\right)+A_{\mathcal{M}} \overrightarrow{v_{m}}\right\} .
\end{aligned}
$$

Each LUT value is obtained from the covering function (16):

$$
\operatorname{Lut}_{\overrightarrow{v_{i}}}[r]=1+\mathcal{C}_{B(O, r-1)}\left(O+\overrightarrow{v_{i}}\right)=1+\max \left(\widehat{\mathcal{H}}(B(O, r-1))+A_{\mathcal{M}} \cdot \overrightarrow{v_{i}}\right) \text {. }
$$

\subsection{LUT Mask}

The algorithm starts with an empty mask and balls with increasing radii are tested for direct covering relations as in [1]. However, in our case, the covering relations are seen from the perspective of the covered balls (in the covering function) whereas in [1], they are considered from the point of view of the covering balls (in the distance map). Another difference lies in the computation of covering radii which does not require the propagation of weights thanks to a direct formula (16). In order to remove useless points, all known LUT neighborhoods are checked for an indirect covering by the procedure visitPoint.

In each influence cone $\left(O,\left\{\overrightarrow{v_{i}}, \overrightarrow{v_{i+1}}\right\}\right)$, a set of covering cones are detected to limit the search space: a $2 \mathrm{D}$ covering cone $((a, a),\{(1,0),(0,1)\})$ with a vertex chosen on the cone bissector $[O,(1,1))$, then $1 \mathrm{D}$ covering cones $((b, \alpha),\{(1,0)\})$, $((\alpha, b),\{(0,1)\})$ for $\alpha$ varying from 1 to $a-1$ (coordinates relative to $\left(O,\left\{\overrightarrow{v_{i}}\right.\right.$, $\left.\left.\overrightarrow{v_{i+1}}\right\}\right)$ ). Fig. 2 shows the working sub-space partitioning, covering cones and visit order of points for the chamfer norm $d_{14,20,31,44}$ and the inner radius 20 . 


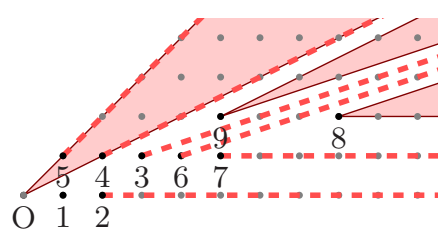

Fig. 2. 1D (dashed lines) and 2D (filled areas) covering cones and visit order of points
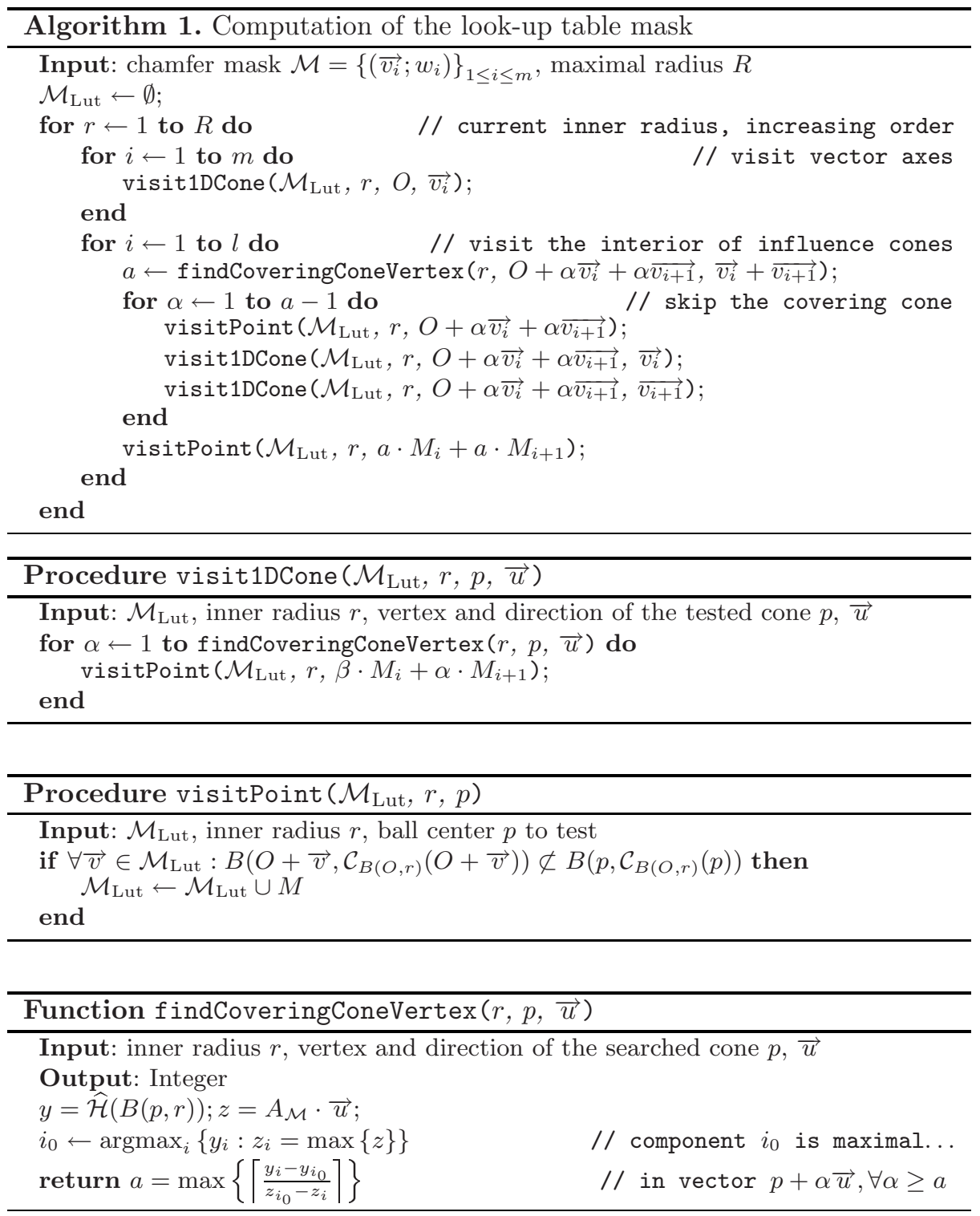
Table 1. Run times (in seconds)

\begin{tabular}{|c|c|c|c|c|c|c|c|c|}
\hline $\mathrm{L}$ & \multicolumn{2}{|c|}{$5,7,11$} & \multicolumn{2}{c|}{$14,20,31,44$} & \multicolumn{2}{c|}{$62,88,139,196,224$} & \multicolumn{2}{l|}{$\begin{array}{l}68,96,152,215,245, \\
280,314,346,413\end{array}$} \\
\hline \hline & reference & $\mathcal{H}$ & reference & $\mathcal{H}$ & reference & $\mathcal{H}$ & reference & $\mathcal{H}$ \\
\hline 200 & 0.468524 & 0.001109 & 1.388558 & 0.005708 & 6.366711 & 0.053448 & 8.867095 & 0.346118 \\
\hline 500 & 8.315003 & 0.002784 & 25.262300 & 0.017302 & 125.293637 & 0.145683 & 177.506492 & 0.975298 \\
\hline 1000 & 90.670806 & 0.007007 & 268.807796 & 0.036611 & 1267.910045 & 0.276737 & 1684.583989 & 1.778505 \\
\hline
\end{tabular}

\subsection{Results}

An implementation of these algorithms was developed in $\mathrm{C}$ language. It produces output in the same format as the reference algorithm [16] so that outputs can be compared character-to-character. Tests were done on various chamfer masks and different maximal radii. The results are almost always identical except for insignificant cases close to the maximal radius for which covering radii exceed the maximum. Other differences may occur in the order weightings are added to $\mathcal{M}_{\text {Lut }}$ (sorted with respect to the covered radius or to the covering radius).

The run times of both reference and proposed algorithms are given in Table 1 for various sizes an distances.

\section{Conclusion and Future Works}

In this paper methods to compute both the chamfer LUT and chamfer LUT mask were presented. Speed gains from the reference algorithm [1] are attributable to the representation of chamfer balls as $\mathcal{H}$-polytopes. This description allows to avoid the use of weight propagation in the image domain and permits a constant time covering test by the direct computation of covering radii. Although not thoroughly tested, we think that LUT value computation is faster with our method due to the smaller size of the test space (linear with the radius of the maximal ball). For $\mathcal{M}_{\text {Lut }}$ determination, results show must faster computation especially for large radii. This is due to the reduced search space eliminating covering cones and the constant time covering test.

While applications always using the same mask can use precomputed $\mathcal{M}_{\text {Lut }}$ and LUT, other applications that potentially use several masks, adaptive masks, variable input image size can benefit from these algorithms. A faster computation of $\mathcal{M}_{\text {Lut }}$ is also highly interesting to explore chamfer mask properties. Beyond improved run times, the $\mathcal{H}$-polytope representation helped to prove new properties of chamfer masks. And a new formula of distance which doesn't need to find in which cone lies a point was given.

Whereas the underlying theory $(\mathcal{H}$-representation of balls, translation, covering test and covering cone) does not depend on the dimension, the algorithms were given only for dimension 2 . In the $2 \mathrm{D}$ case, vectors can be ordered by angle so two consecutive vectors define a cone. Higher dimensions require a Farey triangulation of chamfer balls. 
A paper will greater details and results is being prepared for presentation in a journal. The source code for algorithms presented here is available from the IAPR technical committee on discrete geometry (TC18) 1 .

\section{References}

1. Rémy, É., Thiel, É.: Medial axis for chamfer distances: computing look-up tables and neighbourhoods in 2d or 3d. Pattern Recognition Letters 23(6), 649-661 (2002)

2. Ziegler, G.M.: Lectures on Polytopes (Graduate Texts in Mathematics). Springer, Heidelberg (2001)

3. Rosenfeld, A., Pfaltz, J.L.: Sequential operations in digital picture processing. Journal of the ACM 13(4), 471-494 (1966)

4. Borgefors, G.: Distance transformations in arbitrary dimensions. Computer Vision, Graphics, and Image Processing 27(3), 321-345 (1984)

5. Coeurjolly, D., Montanvert, A.: Optimal separable algorithms to compute the reverse euclidean distance transformation and discrete medial axis in arbitrary dimension. IEEE Transactions on Pattern Analysis and Machine Intelligence 29(3), 437-448 (2007)

6. Strand, R.: Weighted distances based on neighborhood sequences. Pattern Recognition Letters 28, 2029-2036 (2007)

7. Borgefors, G.: Centres of maximal discs in the 5-7-11 distance transforms. In: Proc. 8th Scandinavian Conf. on Image Analysis, Troms $\varnothing$, Norway (1993)

8. Rosenfeld, A., Pfaltz, J.L.: Distances functions on digital pictures. Pattern Recognition Letters 1(1), 33-61 (1968)

9. Thiel, É.: Géométrie des distances de chanfrein. In: mémoire d'habilitation à diriger

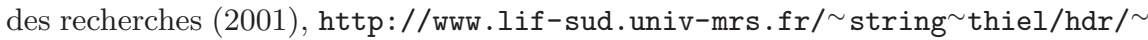

10. Verwer, B.J.H.: Local distances for distance transformations in two and three dimensions. Pattern Recognition Letters 12(11), 671-682 (1991)

11. Rémy, É.: Normes de chanfrein et axe médian dans le volume discret. Thèse de doctorat, Université de la Méditerranée (2001)

12. Thiel, É.: Les distances de chanfrein en analyse d'images: fondements et applications. Thèse de doctorat, Université Joseph Fourier, Grenoble 1 (1994), http://www.lif-sud.univ-mrs.fr/ thiel/these/

13. Pfaltz, J.L., Rosenfeld, A.: Computer representation of planar regions by their skeletons. Communications of the ACM 10(2), 119-122 (1967)

14. Arcelli, C., di Baja, G.S.: Finding local maxima in a pseudo-Euclidian distance transform. Computer Vision, Graphics, and Image Processing 43(3), 361-367 (1988)

15. Svensson, S., Borgefors, G.: Digital distance transforms in 3d images using information from neighbourhoods up to $5 \times 5 \times 5$. Computer Vision and Image Understanding 88(1), 24-53 (2002)

16. Rémy, E., Thiel, É.: Medial axis for chamfer distances: computing look-up tables and neighbourhoods in 2d or 3d. Pattern Recognition Letters 23(6), 649-661 (2002)

17. Remy, E., Thiel, E.: Computing 3D Medial Axis for Chamfer Distances. In: Nyström, I., Sanniti di Baja, G., Borgefors, G. (eds.) DGCI 2000. LNCS, vol. 1953, pp. 418-430. Springer, Heidelberg (2000)

18. Borgefors, G.: Weighted digital distance transforms in four dimensions. Discrete Applied Mathematics 125(1), 161-176 (2003)

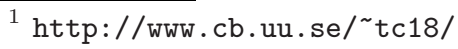

